\title{
Zusammenarbeit von Mensch und Technik im Medienbereich
}

Mit der Digitalisierung geht ein starker Wandel der Arbeitswelt einher. Stichworte sind: Automatisierung, Vernetzung von technischen Geräten („Internet der Dinge"), Künstliche Intelligenz oder Assistenzsysteme. Der vorliegende Beitrag beleuchtet die Effekte der digitalen Transformation auf die Arbeitswelt in der Medienbranche. Anhand verschiedener Beispiele wird aufgezeigt, welch positive Effekte die zukünftige Mensch-Maschine-Kooperation auslösen und wie eine gelungene Gestaltung der Mensch-TechnikOrganisation aussehen kann.

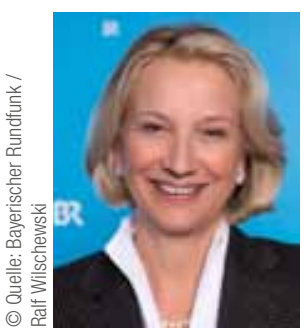

Prof. Dr. phil. Dr.-Ing. Birgit Spanner-Ulmer

Bayerischer Rundfunk

Direktorin Produktion und Technik

Birgit.Spanner-Ulmer@br.de

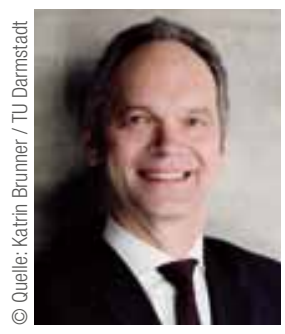

Prof. Dr.-Ing. Ralph Bruder

Technische Universität Darmstadt Institut für Arbeitswissenschaft Bruder@iad.tu-darmstadt.de

Schlüsselbegriffe: Digitale Transformation I Mensch-Technik-Kooperation I Bayerischer Rundfunk

\section{Einführung}

Die Folgen der zunehmenden Digitalisierung sind allgegenwärtig und haben auch zu einem starken Wandel in der Arbeitswelt geführt. Kennzeichnend für diesen Wandel ist ein hoher Vernetzungsgrad von technischen Geräten untereinander („Internet der Dinge“), aber auch zwischen Menschen und Geräten. Die technischen Möglichkeiten, groBe Datenmengen schnell und kostengünstig aufbereiten sowie verarbeiten und daraus Schlussfolgerungen ziehen zu können, haben zudem einen wesentlichen Einfluss auf aktuelle und zukünftige menschliche Arbeitsbedingungen. So ist beispielsweise eine Zunahme der Automatisierung geistiger Tätigkeiten des Menschen unter der Bezeichnung Künstliche Intelligenz zu beobachten.

Aber nicht nur die (Voll)-Automatisierung ist von Bedeutung für die Zukunft menschlicher Arbeit. Insbesondere die Entwicklung und der Einsatz von Assistenzsystemen bietet vielfältige Möglichkeiten zur Unterstützung menschlicher Tätigkeiten. Solche Assistenzsysteme sind gut bekannt in der Automobilbranche (z. B. Parkassistent, ACC; weitere Beispiele vgl. Winner et al. 2015). Aber auch in der Berufswelt haben sich Assistenzsysteme gut etabliert, sei es in der industriellen Arbeit (z. B. Deuse et al. 2015) oder in der Dienstleistungsbranche (z. B. Apt et al. 2018).
Mit der Einführung automatisierter Systeme und noch mehr bei der Nutzung von Assistenzsystemen kommt es zu neuen Formen der Mensch-Technik-Kooperation, wodurch technische Arbeitsvorgänge schneller und sicherer werden können oder wodurch die Verfügbarkeit sowie die Qualität von Dienstleistungsangeboten erhöht werden können. Von Mayer (2018) wurden Möglichkeiten aufgezeigt, wie sich Geschäftsmodelle von Medienunternehmen durch den Einsatz von maschinellem Lernen bei der Wertschaffung, dem Wertversprechen und der Wertschöpfung verbessern lassen. Dazu gehört beispielsweise die Individualisierung von Produkten oder die Kostenoptimierung des Kundenservices durch entsprechende Algorithmen.

Die Medienbranche zählt aber nicht erst in jüngster Zeit zu den Vorreitern der Digitalisierung. Ob in der Produktion (z. B. IP-basierte production, digitale Kameras) oder der Distribution von Content (z. B. digitale Netze) - die digitale Technik hat bereits seit längerem Einzug gehalten (z. B. vbw, 2017).

Die Produktionsprozesse in den Medien lassen sich durch den kreativen Prozess des Erstellens von Content und dem systematischen Zusammenwirken der technischen Systeme charakterisieren. Sie gliedern sich nach Krömker und Klimsa (2005) in folgende Produktionsschritte: 
- Preproduktion: Recherchieren, Planen und Erzeugen von Content;

- Produktion: Anpassen von Content auf das jeweilige Vermittlungssystem - Fernsehen, Hörfunk, Online etc.);

- Postproduktion: Verfeinern, Bearbeiten und Testen von Content;

- Distribution: den Zielkunden Content zur Verfügung stellen.

Immer leistungsfähigere Geräte, hochtechnisierte Datenübertragung und die Möglichkeiten der Verarbeitung groBer Datenmengen lassen die Content-Erstellung mit der technischen Unterstützung immer mehr verschmelzen. Um den Herstellprozess effektiver, effizienter und komfortabler zu gestalten, bietet sich zunehmend der Einsatz neuer Technologien sowohl bei der Content-Generierung als auch bei den technischen Systemen an.

Die Automatisierung ist dabei ein wesentlicher Bestandteil, da sie durch geringeren Zeitaufwand bzw. reduzierten Personaleinsatz hilft, Kosten zu sparen. Zu den weiteren Vorteilen gehört die Qualitätserhöhung durch Fehlerreduktion, das erleichterte Erkennen von Fälschungen oder auch das Ermöglichen von neuen oder verbesserten Mediendiensten und Serviceangeboten.

Automatisierung ist grundsätzlich Bestandteil jeder vernetzten Produktionsumgebung und wird heute an vielen Stellen im Produktionsprozess bereits eingesetzt. Beispiele hierfür sind Programmplanungs-, Produktions- und Automationssysteme sowie Monitoring- und Überwachungssysteme. Herausforderungen liegen nach wie vor in der Automatisierung von Programmplanungs- und Produktionsprozessen über möglichst umfangreiche Prozessketten hinweg.

\section{Abb. 1: Der MT0-Prozess als gleichzei- tige und kontinuierliche Beachtung der Wechselwirkungen von Mensch - Technik - Organisation}

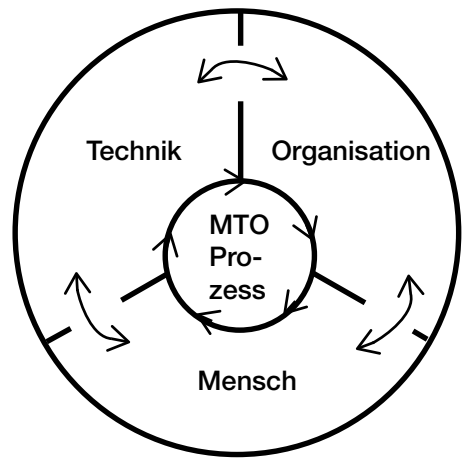

Quelle: Eigene Abbildung
Mit diesem Beitrag soll anhand der Medienbranche aufgezeigt werden, wie eine zukünftige Mensch-Technik-Kooperation so entwickelt und dann auch in Medienunternehmen eingeführt werden kann, damit es zu positiven Effekten kommt.

Als positive Effekte sind zu nennen: höhere Qualität, erweiterte Angebote für Zuschauer/Hörer und Nutzer, Kostenersparnis, höhere Motivation von Mitarbeitenden.

\section{Zusammenwirken von Mensch - Technik - Organisation}

Wichtig für einen gelingenden, $d$. h. effektiven, effizienten und akzeptierten Einsatz von Technik ist die Beachtung des Zusammenhangs von Mensch, Technik und Organisation (MTO) (vgl. Abbildung 1).

Von den Arbeitspsychologen Oliver Strohm und Eberhard Ulich wurde der MTO-Ansatz als Analyse- und Gestaltungskonzept entwickelt (Strohm/Ulich 1997), bei dem Mensch, Technik und Organisation in ihrer jeweiligen Abhängigkeit und ihrem Zusammenwirken zu betrachten sind (Ulich 2011). Eine zentrale Rolle kommt in diesem MTOKonzept nach Strohm und Ulich der Arbeitsaufgabe zu, denn „die Arbeitsaufgabe verknüpft einerseits das soziale mit dem technischen Teilsystem, sie verbindet andererseits den Menschen mit den organisationalen Strukturen" (Ulich 2011). Mithilfe des Verfahrens der MTO-Analyse kann eine ganzheitliche Analyse von Unternehmen auf den Ebenen Unternehmen, Organisationseinheit, Gruppe und Individuum durchgeführt werden (Ulich 2011).

Der Technikwandel, der in erster Linie durch die Digitalisierung getrieben wird, hat Auswirkungen auf die arbeitenden Menschen und die (Arbeits-)Organisation. Daher ist gerade auch im Rahmen der Einführung neuer Technologien das Zusammenwirken von Mensch, Technik und Organisation zu beachten.

Insbesondere sind die Schnittstellen zwischen diesen drei Perspektiven wichtig. So ist beispielsweise bei der Einführung neuer Technologien zu klären, wie sich Organisationen durch Technik verändern. Weiterhin müssen Mensch und Technik aufeinander abgestimmt werden, u. a. durch entsprechende Qualifizierungsmaßnahmen. Schließlich ist auch zu beachten, was sich für einzelne Menschen in der Organisation durch den Einsatz neuer Technologien ändert.

\section{Auswirkungen auf den Menschen:}

Körperliche und monotone Tätigkeiten und einfache Tätigkeiten wie Steuerungsaufgaben können zukünftig vermehrt von intelligenten Maschinen ausgeführt werden. Dies bedeutet, den Wegfall bestimmter Arbeitsplatzprofile. 
Zugleich steigt der Bedarf an Arbeitsplätzen, vor allem an den Schnittstellen zwischen Ingenieursaufgaben und IT-Aufgaben z. B. für IT-Sicherheit. Dies bedeutet eine zunehmende Spreizung der Qualifizierung in entweder sehr einfache Tätigkeiten, also Hilfstätigkeiten oder in hoch qualifizierte Aufgaben, eben die eines Informatikers oder Ingenieurs. Von eben diesen haben wir leider heute schon zu wenige.

Die Anforderungen ändern sich. Entsprechend müssen neue Berufsbilder und Maßnahmen zur Weiterqualifikation entwickelt werden. Das bedeutet Bildung und Qualifikation für die Mitarbeiter.

Die Bereitschaft zu lebenslangem Lernen sowie zum kontinuierlichen Wandel wird von den Menschen in Zukunft noch mehr erwartet werden und auch die Führungsstruktur und die Führungskultur müssen sich anpassen.

\section{Auswirkungen auf die Organisation:}

In den bisherigen Ausführungen wurde insbesondere die Koexistenz bzw. Kooperation zwischen Technik und Mensch betrachtet. Das Bindeglied zwischen beiden ist in Unternehmen die (Arbeits-) Organisation.

Im Hinblick auf die Organisation müssen innovative Beschäftigungsmodelle entwickelt werden. Beispielsweise müssen IT-Spezialisten nicht mehr zwingend vor Ort im Unternehmen sein. Sie können evtl. von einem Telearbeitsplatz aus agieren. Das spart dem Unternehmen Fläche in Form eines Dauerarbeitsplatzes, und dem Mitarbeiter eröffnet es die freie Gestaltung des Arbeitstages.

Dies erfordert wiederum andere und neue Arbeitszeitmodelle. War bisher die Anwesenheit im Unternehmen eine Messgröße, könnte dies in Zukunft durch die Tätigkeitszeit oder die Vertrauensarbeitszeit abgelöst werden.

Veränderungen in Prozessen und Arbeitsabläufen werden notwendig. Dies kann bei den Mitarbeitern zur Verunsicherung führen. Insbesondere die Führungskräfte sind hier gefordert und benötigen Unterstützung.

Als Folge all dieser Entwicklungen werden Arbeitszeit und Freizeit zunehmend verschmelzen. Dies wirft weitere Fragen für die digitale Gesellschaft auf:

Muss jede Mitarbeiterin, jeder Mitarbeiter jederzeit erreichbar sein? Eine solche kontinuierliche Erreichbarkeit bei gleichzeitig hohem Termin- und Leistungsdruck kann auf Dauer zu Stress führen, und Stress ist eine häufige Krankheitsursache (BAuA, 2016).
Manche Firmen haben dieses Problem erkannt und mit einem Verbot von Mail-Antworten nach bestimmten Uhrzeiten (Medienunternehmen mit 24h-Betrieb?) bereits gelöst. Für die Zukunft ist diese Vorgehensweise allerdings nur eingeschränkt tragfähig. Es muss vielmehr der Umgang mit der ständigen Erreichbarkeit - z. B. was den Gebrauch der Smartphones anbelangt - erlernt werden im Sinne einer zielgerichteten Nutzung für unseren Alltag. Die eigene Verantwortung im Umgang mit neuen Technologien bedarf einer gewissen Disziplin auf allen Seiten. Das bedeutet eine Veränderung in der Unternehmens- und Führungskultur.

Neben der Betrachtung der einzelnen Schnittstellen von Mensch-Technik-Organisation ist aber eine integrative Gesamtsicht notwendig. Hier gibt es keine zeitliche Abfolge beispielsweise im Sinne: zunächst Technik einführen, dann Menschen schulen und dann die Organisation ändern. Die parallele Betrachtung von technischen Möglichkeiten, menschlichen Kompetenzen und organisationalen Änderungen ist für eine erfolgreiche Einführung neuer Technologien entscheidend.

\section{Mensch-Technik-Kooperation in der Medienbranche am Beispiel des BR}

Der Technologiewandel hat in der Medienbranche weitreichende Auswirkungen. Aus einer Vielzahl von Beispielen werden im Folgenden diejenigen vorgestellt, die im Bayerischen Rundfunk zu bedeutenden Veränderungen gehörten bzw. gehören. Alle Beispiele haben, wie in Kapitel 2 beschrieben, durch die Möglichkeiten der Technik Veränderungen in den organisatorischen Abläufen und bei den Mitarbeitenden zur Folge.

\section{Beispiel 1: Neue Technologien verändern die Archive (Preproduktion)}

Data Science, Analysemethoden und lernende Maschinen schaffen die Möglichkeiten für automatisierte (Nachrichten-)Recherchen und -Erstellung sowie für die assistierte Suche und Analyse von Archivmaterial, bis hin zur Analyse, welche Themen „im Netz" gerade relevant sind.

Durch das sogenannte Data-Mining in Video-, Audio- und Untertitelspuren können automatisiert textuelle Beschreibungen und Inhalte erzeugt werden (automatisierte Gesichts-, Objekt- und Stimmungserkennung, Speech-toText), die wiederum eine deutlich schnellere Recherche auf Textbasis erlauben.

Die Archive des BR setzen Mining-Technologie zur Verwaltung der Archivbestände in den Bereichen Text bzw. Video bereits seit einigen Jahren ein. Presseartikel werden weitestgehend automatisch verschlagwortet, unter- 


\section{Aufsätze}

schiedliche Fassungen von Videos automatisch verglichen und die Segmentierung von Beiträgen deutlich erleichtert. Aktuell laufen Tests mit Videomining, um unter anderem mit automatischer Gesichtserkennung bereits im Produktionsprozess die Redaktionen bei der Materialverwaltung zu unterstützen und die Archivierung bzw. Dokumentation zu erleichtern. Die automatisierte Extrahierung von Videoausschnitten mit Bauchbinden aus den BR-Archiven ist ein erster wichtiger Schritt. Diese Extrakte konnten zum kostengünstigen und wirksamen Training der Algorithmen einer Gesichtserkennungssoftware eingesetzt werden. Das Computerprogramm erkennt nun automatisiert eine Vielzahl bayerischer Landespolitiker.

Seit kurzem nutzt das Archiv auch Audiomining. Eine Software "liest" einen verschrifteten Radiobeitrag und findet im Audio die korrespondierende Stelle. Sie „hört“ sich das Audio an und vergibt selbsttätig Schlagworte und Ressorts für Redaktion und Archiv. Sie erkennt unterschiedliche Sprecher und fügt Audiotakes, etwa Anmoderation und Beitrag, zusammen.

\section{Beispiel 2: Automatisierte Content-Generierung (Preproduktion)}

Mit Hilfe der künstlichen Intelligenz sind Systeme inzwischen in der Lage, Nachrichten (z. B. Wetter, Sportergebnisse) automatisiert zu erstellen. Erstellt von einer Software, die ein Nachrichtenredakteur anwendet und die Ergebnisse auf Plausibilität prüft. Das ermöglicht der Medienanstalt eine sehr schnelle Berichterstattung. Vor allem für News- und Sport-Content eignet sich dieser Produktionsprozess. Textgenerierung wird als Dienstleistung angeboten. Der Kunde bestimmt, wie ein Text aufgebaut sein und sprachlich klingen soll. Gemeinsam mit den Entwicklern der Software wird diese sozusagen trainiert. Um einen Text zu formulieren, muss das System Regeln lernen, die ihm der Mensch beibringt. Bspw. muss er im Fußball erkennen können, dass ein 2:0 Ergebnis normal, ein 10:0 Ergebnis außergewöhnlich ist („automated annotation“).

Die Chancen liegen auf der Hand. Mit wenigen Stichworten und in kurzer Zeit werden Beiträge verfasst. Die positive Sicht dieser Entwicklung ist sehr gut nachzuvollziehen. Warum sollte sich ein Algorithmus, also die automatisierte Textgenerierung, nicht um schnelle News, wie z. B. um Wetter und Verkehr annehmen? In dieser Zeit kann sich der Redakteur um die Einordnung der Nachrichten und um die Hintergründe kümmern. Er oder sie kommentiert, recherchiert und analysiert, indem Erfahrungswissen eingesetzt wird, über das ein Computer nicht verfügt. Befragungen haben ergeben, dass Texte, die von Computern erstellt wurden, kaum von denen von Journalisten zu unterscheiden sind. In diesem Zusammenhang sollte allerdings beachtet werden, dass eine solche automatisierte Content-Generierung bei Autoren und Journalisten nachvollziehbar zu Existenzängsten führt. Dies ist bei der Einführung solcher Automatisierungsansätze dringend zu berücksichtigen (vgl. Kapitel 2).

\section{Beispiel 3: Automatisierung durch Kameraro- boter am Beispiel des BR Rundschau-Studios (Produktion)}

In automatisierten TV-Studios kommen Industrieroboter zum Einsatz. Sie ersetzen als Träger der Studiokameras die klassischen Kameraleute. Alle Kameraeinstellungen und -fahrten werden aus dem Redaktionssystem gesteuert. Eine vollautomatisierte Sendung benötigt im ungestörten Sendeablauf dafür kein menschliches Eingreifen mehr (Perspektive Technik).

Bei der Erneuerung des Studios für die BR Rundschau war dies die Vision. Während die bisherigen Rundschausendungen von drei Kameraleuten direkt im Studio aufgenommen wurden, stehen im neuen Studio nun ein Kameraroboter und zwei fernsteuerbare Robotikstative, deren Fahrten vorab fest einprogrammiert werden können. Sie werden vom benachbarten Regieraum aus per Joystick bedient oder direkt von der Automation angesteuert. Ein EchtzeitGrafiksystem erlaubt es, dass die Grafiken bereits vor der Sendung in den Ablauf eingebaut und vom Computer automatisch ausgespielt werden können. Moderatorinnen oder Moderatoren sind allein im Studio. Kein Kameramann, Teleprompter oder Studiooperator ist dort mehr zu finden. In der Mitte des virtuellen Studios befindet sich als einziges reales Element ein Moderationspult mit einer geschwungenen Platte. Jede Kamera im Studio ist mit einem Trackingsystem versehen, und alle Bilder werden in Echtzeit mit Hochleistungsrechnern gerendert.

Die Vorteile einer solchen Automatisierung sind offensichtlich. Mit geringerem personellem Aufwand lässt sich die Sendung sehr gut fahren. Allerdings verändert sich für die Kameraleute die Tätigkeit - sie müssen z. B. als StudioOperator Neues lernen (Perspektive Mensch).

Auch für den Moderator ist dies eine ungewohnte und damit gewöhnungsbedürftige Situation, beispielsweise durch das Einhalten von Sicherheitsabständen zum Roboter (Perspektive Mensch).

Zur Sicherheit wurden einige Sensoren so im Studio verbaut, dass niemand sich dem Roboterarm nähern kann, ohne Gefahr durch automatisierte Kamerabewegungen verletzt zu werden. Würde der Sicherheitsbereich betreten werden, so schalten sich umgehend alle Robotiksysteme in Stand-By und können nur vom Studio-Operator in der Regie wieder freigegeben werden. 
Der große technische Aufwand, der für die Umsetzung dieses neuen Studios bewältigt werden musste, war deshalb so erfolgreich, weil Redaktion und Technik von Anbeginn der Planung bis zur Umsetzung im Sinne des MT0-Prozesses sehr eng zusammengearbeitet haben. Damit sind Verständnis aufeinander und Vertrauen ineinander gewachsen.

\section{Beispiel 4: Smart Production (Produktion)}

In diesem Zusammenhang wird unter „smart production“ verstanden, mit skalierbaren Produktionsmitteln/Technik für die jeweiligen Anforderungen möglichst günstige und angepasste Lösungen anzubieten. Ziel ist, mit weniger Personal den gleichen Content und damit kostengünstiger zu produzieren.

Ein Beispiel für smart production ist die sogenannte „Subregie" im Rahmen des neu installierten BR Rundschau-Studios. Sie ist insbesondere für Social Media-Anwendungen wichtig. Mit inr können parallele Ausspielungen vorgenommen werden bzw. als Livestream eine zweite Sendestrecke zum linearen BR Fernsehen aufgebaut werden. Mit der Subregie können sechs Kanäle, wie Facebook, Twitter etc. bespielt werden.

Der dazugehörige Schaltenplatz ist so gestaltet, dass ein Moderator alleine in der Lage ist, diesen Platz zu bedienen. An einem höhenverstellbaren Tisch wurde eine Kamera mit entsprechender Beleuchtung angebracht. Gerade im Breaking-News-Fall ist der Moderator so in der Lage, sofort Informationen über diese unterschiedlichen Kanäle auszuspielen (siehe Abbildung 3).

\section{Abb. 2: Studio für BR Rundschau mit Kamera- robotern}

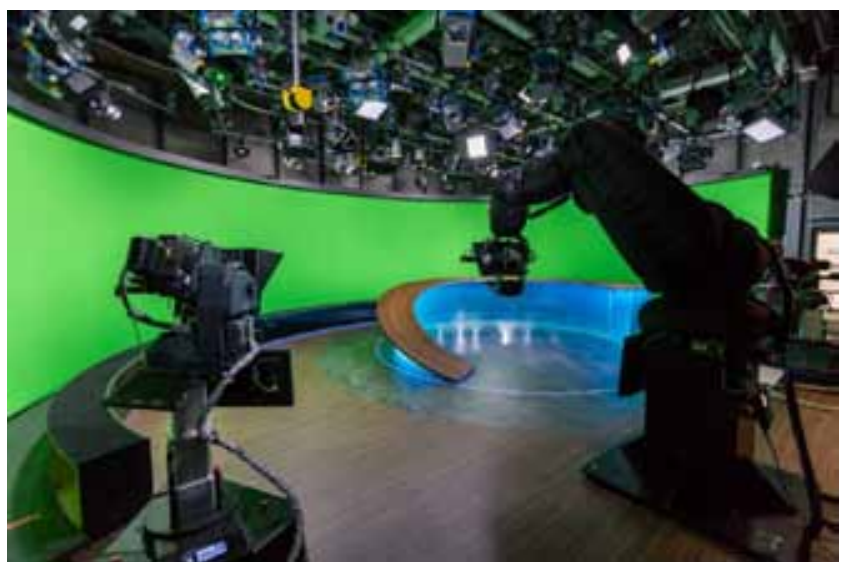

Quelle: Bayerischer Rundfunk
Der „Technologiesprung“ lässt sich auch anhand eines anderen Beispiels belegen. Um die bayerischen Beachvolleyball-Meisterschaften der Damen per Streaming zu übertragen, kam das BR Weblink-Fahrzeug zum Einsatz. Das Fahrzeug ist technisch hoch innovativ ausgestattet, um in erster Linie Liveinhalte für Onlineplattformen, wie z. B. BR24, herzustellen. Ausgerüstet mit mehreren iPhones oder wahlweise anderen Kameratypen, wie GoPro, die über ein internes WLAN oder LTE-Mobilfunk drahtlos mit dem Weblink verbunden sind, können diese Bildquellen im Fahrzeug live gemischt werden. In einem definierten Rahmen können auch Grafikeinblendungen vorgenommen werden. Die Aufgabe speziell bei dieser Sportübertragung war, dauerhaft den aktuellen Spielstand in das Sendesignal einzublenden, ohne dass weiterer Personaleinsatz während der Produktion notwendig würde. Gelöst wurde dies durch ein Grafiktemplate im BR-Sportdesign, das vor der Produktion im Grafiksystem im Weblink angelegt wurde. BR-Kollegen programmierten ein kleines Tool, das den aktuellen Spielstand von der Webseite des Beachvolleyballverbandes ausgelesen und an das Grafiktemplate übergeben hat. Damit konnte der Spielstand grafisch ansprechend aufbereitet und ständig aktuell automatisiert eingeblendet werden. Solche intelligenten Lösungen sind Vorbild für weitere Entwicklungen.

\section{Beispiel 5: Mobile und non-lineare Angebote (Distribution)}

Die starke Veränderung der technischen Möglichkeiten ist auch verbunden mit einer deutlichen Veränderung der Nutzergewohnheiten, insbesondere der 14- bis 29-Jährigen. Der Abbildung 4 ist zu entnehmen, dass lineares Programm (noch) stark genutzt wird. So beläuft sich die Nutzungsdau-

\section{Abb.3: Assistierte Arbeitsumgebung für den Selbstfahrer am Moderationsplatz}

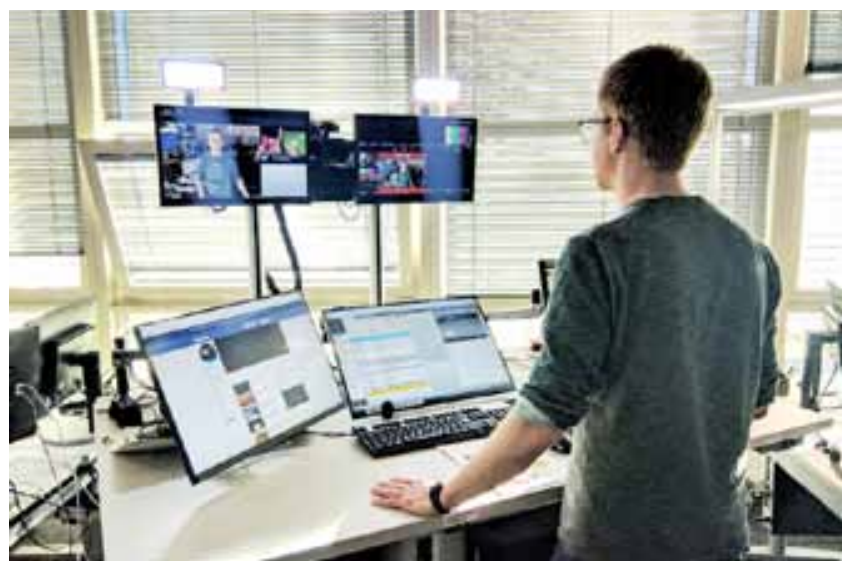

Quelle: Bayerischer Rundfunk 


\section{Abb. 4: Nutzungsentwicklung Fernsehen, Radio, Internet 2002 bis 2018, tägliche Nutzungsdauer}
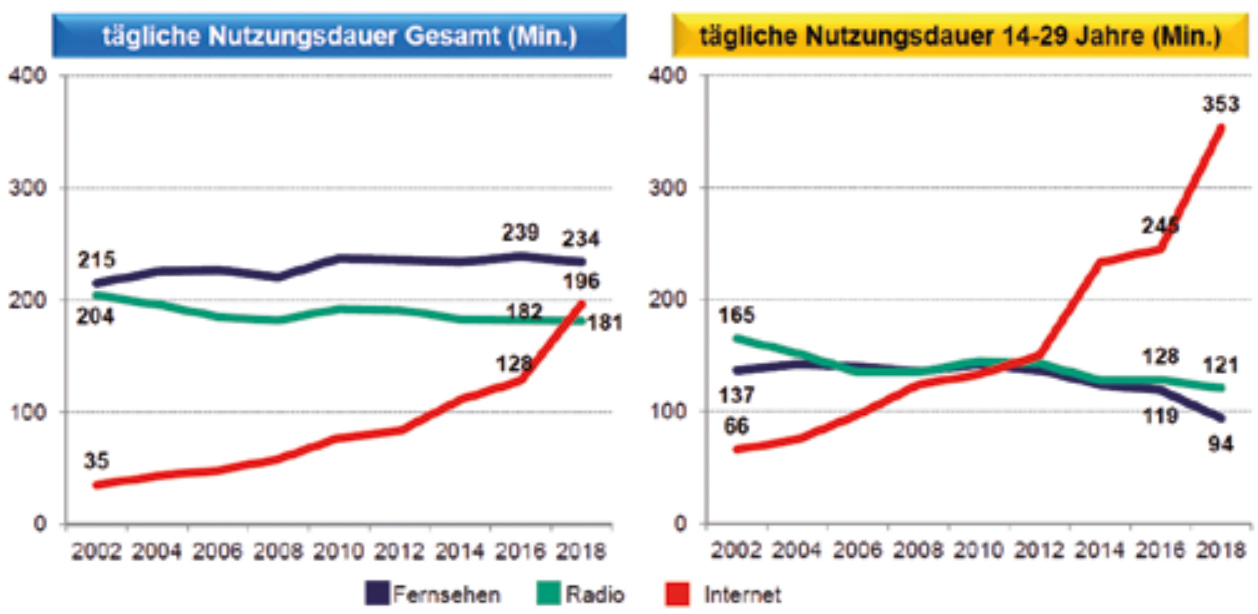

1 gber ein stationares TV.Gerats ofhe: Live.

2 unsthingig vom Vertoreitungeseneg

Basis: Erwachsene ab 14Jahre in Deutschland, Montag bis Sonntag

er für Fernsehen auf 234 Minuten täglich, im Hörfunk sind es 181 Minuten. Die Nutzungsdauer des Fernsehens ist in den vergangenen 16 Jahren sogar gewachsen. Gleichzeitig ist ein massives Wachstum in der Nutzung von Onlineangeboten zu verzeichnen. Überall, von jedem Ort und zu jeder Zeit Programm abrufen zu können, wird von vielen Nutzern geschätzt. Mobiles und non-lineares Angebot erfährt damit zunehmend an Bedeutung. Mit den Möglichkeiten des Internets werden insbesondere von der Gruppe der 14- bis 29-Jährigen Plattformen wie Amazon Netflix, Spotify und Social-Media-Plattformen wie Facebook, Twitter oder Instagram genutzt.

Um den Erwartungen des Publikums gerecht zu werden, setzt der BR verstärkt auf Online-Programmangebote. Dazu gehören neben der Übertragung des linearen Angebots via Internet eigene Formate der Social-Media-Kanäle, die BR24 App, sowie die BR Mediathek. Diese wurde vor kurzem nutzergerecht weiterentwickelt. Filme, Serien und Dokumentationen werden in den Vordergrund gestellt. Das Neue ist: In manchen Bereichen der Mediathek werden Videos angeboten, die speziell auf Sehgewohnheiten und Interessen des Nutzers abgestimmt sind. Die MediathekRedaktion kuratiert und stellt Highlights und aktuelle Videos auf der Startseite nach Rubriken sortiert zusammen. Ein spezieller Vorteil ist, dass der Nutzer ein eigenes Konto anlegen kann, um darüber Sendungen zu abonnieren, die inn interessieren. Automatisiert werden Videoempfehlungen angeboten, und das sogenannte „seamless viewing" kann genutzt werden. Darunter versteht man, dass sobald ein Video auf einem Gerät gestartet wurde, es auf einem anderen Gerät weiter genutzt werden kann.

In der folgenden Tabelle 1 werden die dargestellten Fallbeispiele des Bayerischen Rundfunks den einzelnen Produktionsschritten zugeordnet und es wird der jeweilige Umsetzungsstand aufgezeigt.

\section{Folgerungen}

Die Digitalisierung verändert die Welt in einer nie da gewesenen Dynamik. Die damit verbundene Transformation in allen gesellschaftlichen Bereichen muss jederzeit mitgedacht werden.

Wie die Beispiele zeigen, bietet die Nutzung des MT0Ansatzes gute Chancen zu einer erfolgreichen Einführung neuer Technologien. Folgende Thesen lassen sich aus den Beispielen ableiten und auf ähnliche Fragestellungen übertragen:

(1) Neue Technologien in der Medienbranche haben das Potential, die Effizienz von Arbeitsabläufen zu erhöhen, die Qualität des Content zu verbessern und damit auch den Kundennutzen zu steigern. Eine gleichzeitige und gleichrangige Optimierung aller drei Kriterien (Effizienz, Qualität und Kundennutzen) gelingt allerdings nur selten. Es gilt 


\section{Tab. 1: Beispiele für Mensch-Technik-Kooperation in verschiedenen Produktionsphasen beim BR}

\begin{tabular}{lll} 
Beispiel & Produktionsschritt & Umsetzungsstand \\
\hline Veränderung in den Archiven & Preproduktion & In Entwicklung \\
\hline Automatisierte Content-Generierung & Preproduktion & In Entwicklung \\
\hline Kameraroboter im BR Rundschau-Studio & Produktion & Im Einsatz \\
\hline Smart Production & Produktion & Im Einsatz \\
\hline Mobile und non lineare Angebote & Distribution & Im Einsatz und weiterer Entwicklung \\
\hline
\end{tabular}

daher vor Beginn der Einführung neuer Technologien, Prioritäten hinsichtlich der zu erreichenden Ziele zu setzen.

(2) Die Abstimmung zwischen technologischen Veränderungen, deren organisatorischen Folgen und den menschbezogenen Auswirkungen (u. a. Akzeptanz, Kompetenzerwerb) ist notwendige Voraussetzung für eine gelingende Einführung neuer Technologien. Hier ist die Festlegung eines jeweils projektspezifischen MT0-Prozesses notwendig, mit der Berücksichtigung der Expertise in den Bereichen Mensch, Technik, Organisation.

(3) Eine wesentliche Bedingung für das Gelingen neuer Technologieeinführungen ist eine nutzungsfreundliche Mensch-Technik-Kooperation. In diesem Zusammenhang hat sich die Beachtung des menschzentrierten Entwicklungsprozesses nach ISO 9241 Teil 210 (2011) bewährt. Konsequent wäre also zu fordern, dass nur solche MenschTechnik-Schnittstellen zum Einsatz kommen, bei denen ein solcher menschzentrierter Entwicklungsprozess auch nachweisbar stattgefunden hat.

(4) Mit einer nutzungsfreundlichen Mensch-Technik-Interaktion führen neue Technologien häufig auch zu neuen Anforderungen an die Kompetenz von Nutzerinnen und Nutzern. Erst durch entsprechende Kompetenzen können die Funktionalitäten und möglichen Arbeitserleichterungen in vollem Umfang genutzt werden. Eine Bereitschaft zur Weiterbildung auf Seiten der Mitarbeiterinnen und Mitarbeiter ist deshalb eine weitere notwendige Bedingung für das Gelingen der Einführung neuer Technologien. Hier sind Organisationen gefordert, für eine gute Kultur des lebenslangen Lernens im eigenen Unternehmen u. a. mit entsprechenden Angeboten zu sorgen. Zudem kommt den Führungskräften eine wichtige Rolle in der Förderung der Weiterentwicklung von Kompetenzen der Mitarbeitenden zu.

\section{Literatur}

Apt, W./Schubert, M./Wischmann, S. (2018): Digitale Assistenzsysteme, Institut für Innovation und Technik (iit), Berlin.

BAuA (2016): Arbeitszeitreport Deutschland 2016, Dortmund (Bundesanstalt für Arbeitsschutz und Arbeitsmedizin).

Deuse, J./Weisner, K./Hengstebeck, A./Busch, F. (2018): Gestaltung von Produktionssystemen im Kontext von Industrie 4.0, in: Botthof, A./Hartmann, E. A (Hrsg.): Zukunft der Arbeit in Industrie 4.0, Wiesbaden, S.99-109.

ISO 9241 Teil 210 (2011): Ergonomie der Mensch-Maschine-Interaktion - Teil 210: Prozess zur Gestaltung gebrauchstauglicher interaktiver Systeme, Ber-

Krömker, H./Klimsa, P. (2005): Einführung, in: Krömker, H./Klimsa, P. (Hrsg.) Handbuch Medienproduktion, Wiesbaden, S. 15-36.

Mayer, C. (2018): Künstliche Intelligenz und Maschinelles Lernen: Hintergrund, Anwendungsfälle und Chancen für Medienunternehmen, in: MedienWirtschaft, 15. Jg., H. 3, S.30-35.

Strohm, 0./Ulich, E. (Hrsg.)(1997): Unternehmen arbeitspsychologisch bewer ten: Ein Mehrebenenansatz unter besonderer Berücksichtigung von Mensch Technik und Organisation, Zürich (Schriftenreihe Mensch - Technik - Organisation, Band 10)

Ulich, E. (2011): Arbeitspsychologie, 7. Aufl., Stuttgart.

vbw (Hrsg.)(2017): Medienstandort Bayern - Technologie schafft Profil, München.

Winner, H./Hakuli, S./Lotz, F./Singer, C. (Hrsg.)(2015): Handbuch Fahrerassistenzsysteme, Wiesbaden 